\title{
Search for exoplanet around northern circumpolar stars
}

\section{Four planets around HD 11755, HD 12648, HD 24064, and 8 Ursae Minoris $\star$}

\author{
B.-C. Lee (이병 철 $)^{1,2}$, M.-G. Park (박명구 $)^{3}$, S.-M. Lee (이상민 $)^{1}$, G. Jeong (정 광희 $)^{1,2}$, H.-I. Oh (오형 일 $)^{3}$, \\ I. Han (한인우) ${ }^{1}$, J. W. Lee (이재우) ${ }^{1}$, C.-U. Lee (이충욱) ${ }^{1}$, \\ S.-L. Kim (김승리) ${ }^{1}$, and K.-M. Kim (김강민) ${ }^{1}$
}

\author{
1 Korea Astronomy and Space Science Institute, 776, Daedeokdae-Ro, Youseong-Gu, 305-348 Daejeon, Korea \\ e-mail: bclee@kasi.re.kr \\ 2 Korea University of Science and Technology, Gajeong-ro Yuseong-gu, 305-333 Daejeon, Korea \\ ${ }^{3}$ Department of Astronomy and Atmospheric Sciences, Kyungpook National University, 702-701 Daegu, Korea
}

Received 29 July 2015 / Accepted 24 September 2015

\section{ABSTRACT}

\begin{abstract}
Aims. This program originated as the north pole region extension of the established exoplanet survey using the $1.8 \mathrm{~m}$ telescope at Bohyunsan Optical Astronomy Observatory (BOAO). The aim of our paper is to find exoplanets in northern circumpolar stars with a precise radial velocity $(\mathrm{RV})$ survey.

Methods. We selected about 200 northern circumpolar stars with the following criteria: $\delta \geq 70^{\circ}, 0.6<B-V<1.6$, HIPPARCOS $_{\text {scat }}<0.05 \mathrm{mag}$, and 5.0 $<m_{v}<7.0$. The high-resolution, fiber-fed Bohyunsan Observatory Echelle Spectrograph (BOES) was used for the RV survey. Chromospheric activities, the HIPPARCos photometry, and line bisectors were analyzed to exclude other causes for the RV variations.

Results. In 2010, we started to monitor the candidates and have completed initial screening for all stars for the past five years. We present the detection of four new exoplanets. Stars HD 11755, HD 12648, HD 24064, and 8 UMi all show evidence of giant planets in Keplerian motion. The companion to HD 11755 has a minimum mass of $6.5 M_{\text {Jup }}$ in a 433-day orbit with an eccentricity of 0.19 . HD 12648 is orbited by a companion with a minimum mass of $2.9 M_{\text {Jup }}$, a period of 133 days, and an eccentricity of 0.04 . Weak surface activity was suspected in HD 24064. However, no evidence was found to be associated with the RV variations. Its companion has a minimum mass of $9.4 M_{\text {Jup }}$, a period of 535 days, and an eccentricity of 0.35 . Finally, 8 UMi has a minimum mass of $1.5 M_{\text {Jup }}$ and a period of 93 days with an eccentricity of 0.06 .
\end{abstract}

Key words. techniques: radial velocities - planetary systems - stars: individual: HD 11755 - stars: individual: HD 12648 stars: individual: HD 24064 - stars: individual: 8 Ursae Majoris (HD 133086)

\section{Introduction}

Since the 1990s, exoplanet surveys by precise radial velocity (RV) method have been conducted in many observatories around the world. Nearly $25 \mathrm{yr}$ of concerted RV monitoring have revealed interesting and diverse planetary systems. Almost all initial exoplanet search programs have focused on G, K main sequence (MS) as host stars because they are bright enough to have a high signal-to-noise ratio $(\mathrm{S} / \mathrm{N})$ and have an ample number of spectral lines for precise RV measurements. However, due to a deficit of candidate stars at this stage of ground-based observations, searches have gradually been expanded to evolved stars (Frink et al. 2002; Setiawan et al. 2003; Sato et al. 2003; Hatzes et al. 2005; Döllinger et al. 2007; Han et al. 2010). There is no doubt that most planet search programs reflect a bias toward relatively bright stars and against fainter $\mathrm{M}$ dwarfs. However, $\mathrm{M}$ dwarfs are the most numerous class of stars in the solar neighborhood, comprising $~ 75 \%$ of the stars (Reid et al. 2002). Low-mass stars may be the most typical targets of planet formation (Lada 2006), and they were collectively monitored by several groups (Endl et al. 2003; Wright et al. 2004; Bonfils et al. 2005). Now M dwarfs are the most active observational target

\footnotetext{
* Based on observations made with the BOES instrument on the $1.8 \mathrm{~m}$ telescope at Bohyunsan Optical Astronomy Observatory in Korea.
}

(Anglada-Escudé et al. 2012; Bonfils et al. 2013; Howard et al. 2014; Bowler et al. 2015).

Most of the ground-based RV surveys have been conducted in the form of an all-sky survey in accordance with the classification of spectral type and luminosity class and developed to maximize the RV accuracy of a star. But, this strategy causes a bias. Most of RV observatories in the northern hemisphere are focusing observations on specific spectral or luminosity class rather than targeting a specific area. Owing to the geographical constraints and the effective operation of the telescope, high declination zone has not been observed actively. Even in Hawaii where large telescopes for spectroscopic observatories are densely populated, observations of more than $70^{\circ}$ declination are not effective. As a result, this area has become a likely blind spot for planet surveys $\left(+70^{\circ}<\delta<+90^{\circ}\right.$ and $\left.-70^{\circ}<\delta<-90^{\circ}\right)$. Around 20 exoplanets have been found in this region (6\% of the total area), corresponding to $\sim 3 \%$ of detection by the RV method and $\sim 1 \%$ of the total exoplanet detection. Here, we introduce a new exoplanet survey, a "Search for Exoplanet around Northern circumpolar Stars (SENS)", which uses the $1.8 \mathrm{~m}$ telescope at Bohyunsan Optical Astronomy Observatory (BOAO) in Korea to search for planets among several survey projects.

In this paper, we present the first result from the survey. In Sect. 2, we introduce the SENS program. Section 3 describes the 
observational data and reduction procedures. We give the stellar characteristics in Sect. 4 and the RV analysis and the nature of the RV measurements for each star in Sect. 5. Finally, in Sect. 6 we discuss the result from this study.

\section{The SENS program}

Our survey targets are selected from the HIPPARCos catalog (ESA 1997) according to the following criteria: stars with 1) the declination $\delta \geq 70$;2) $5.0<m_{v}<7.0$ to attain a sufficient $\mathrm{S} / \mathrm{N}$, which are barely observable using the $2 \mathrm{~m}$ class telescopes with a Doppler precision of $\sim 10 \mathrm{~m} \mathrm{~s}^{-1}$; 3) a color index of $0.6<B-V<1.6$ to study precise RV measurements with an ample number of spectral lines; and 4) excluding stars identified as known photometric variables or having photometric scatters over 0.05 mag based on the results of the HIPPARCOS photometry. In total, we have selected about 200 stars for the survey, which consist of late-F, G, K, and early M stars covering all luminosity classes. (The fractions in these spectral classes are $2 \%$, $33 \%, 60 \%$, and 5\%, respectively.) Our target list includes two known planetary systems. This overlap will serve as a check on the systematic difference with other RV measurements.

Our survey has three advantages. First, the area was not been actively observed for an exoplanet survey. Second, since the candidates are concentrated around the pole star, this reduces the telescope travel time to the successive target (less than $30 \mathrm{~s}$ ). Finally, observations are possible throughout the year.

Our basic procedure is composed of the following three steps. In the first step, we observe a large number of candidate stars with a $\mathrm{S} / \mathrm{N}$ of $\sim 150$ by 2012 . During this period, observing time is scheduled such that each target should receive four to five observations per year for the first screening to identify stars showing large RV variations. This strategy appears to reduce the probability of detecting shorter-period exoplanets because they require more densely sampled observations. We do, however, note that our sample contains just $5 \%$ of MS stars, which are expected to be slightly more frequently observed. In the second step, we conduct follow-up observations to confirm the periodicity of stars that turn out to show large RV variations, which is more than three times the RV variations of the RV standard star $\tau$ Ceti. This continues for two years. In a final step, we focus on detecting planets with periodicity. We plan to monitor the selected targets during the next two years to cover a complete orbital cycle for planetary candidates. This program takes seven years in total.

\section{Observations and data reduction}

Observations were carried out using the fiber-fed high-resolution Bohyunsan Observatory Echelle Spectrograph (BOES; Kim et al. 2007) attached to a $1.8 \mathrm{~m}$ telescope at BOAO in Korea. Generally, to reduce the line broadening due to a stellar rotation, an exposure time is limited to less than about $20 \mathrm{~min}$ in the precise RV measurements. To obtain an appropriate signal with respect to the candidates $\left(5.0<m_{v}<7.0\right)$, the resolution of the optical fiber of 45000 is most advantageous among three kinds of available resolutions $R=90000,45000$, and 27000 . To obtain precise RV measurements, we used an iodine absorption $\left(\mathrm{I}_{2}\right)$ cell, which superimposes thousands of molecular absorption lines over the object spectra in the spectral region between 4900 and $6100 \AA$. Using these lines as a wavelength standard, we simultaneously modeled the time-variant instrumental profile and Doppler shift relative to an $I_{2}$ free template spectrum.
Observations for the SENS began at BOAO in January 2010. The program has received about 17 nights per year from the beginning, and approximately $40 \%$ has produced usable data. We aim for a $\mathrm{S} / \mathrm{N}$ of $\sim 150$ at $5500 \AA$, resulting in exposure times ranging from 10 to $20 \mathrm{~min}$.

Extraction of the spectra from raw CCD images was carried out using the IRAF software that performs bias correction and flat fielding, thereby removing scattered light from interorder pixels, and subtracts one-dimensional spectra. The precise RV measurements related to the $\mathrm{I}_{2}$ analysis were undertaken using the RVI2CELL (Han et al. 2007), which is based on a method by Butler et al. (1996) and Valenti et al. (1995). The long-term stability of the BOES has been monitored by observing the RV standard star $\tau$ Ceti since 2003. It demonstrates a long-term RV stability of the spectrometer with an rms scatter of about $7 \mathrm{~m} \mathrm{~s}^{-1}$ (Lee et al. 2013).

\section{Stellar characteristics}

\subsection{Fundamental parameter}

The basic stellar parameters were taken from the HIPPARCos catalog (ESA 1997) and the improved parallaxes from van Leeuwen (2007). The distance and luminosity came from the result of Anderson \& Francis (2012). The stellar atmospheric parameters were determined using the TGVIT (Takeda et al. 2005) program based on the Kurucz (1992) atmosphere models. We used more than 150 equivalent width (EW) measurements of $\mathrm{Fe}$ I and Fe II lines for each star. Stellar radii and masses were estimated from the stellar positions in the color-magnitude diagram and with the theoretical stellar isochrones of Bressan et al. (2012). We also adopted a version of the Bayesian estimation method (Jørgensen \& Lindegren 2005; da Silva et al. 2006) by using the determined values for $T_{\mathrm{eff}},[\mathrm{Fe} / \mathrm{H}], m_{v}$, and $\pi$. It also provided the most likely values for stellar age. The basic stellar parameters are summarized in Table 1.

\subsection{Rotational period}

In evolved stars, the stellar rotational period is very important for identifying the RV variation from the rotational modulations of surface structures. The observed stellar spectrum was fitted by convolving the component functions, which broaden spectral lines without altering their EW. Determining the origin of the broadening is difficult for spectra of slowly rotating late type stars because they have similar intrinsic line profiles.

To estimate the stellar rotational velocities, a line-broadening model by Takeda et al. (2008) was used. To determine line broadening, the automatic spectrum-fitting technique (Takeda 1995) for the spectrum within the wavelength range 6080-6089 $\AA$ was also used. We estimated the rotational velocities of $2.3 \mathrm{~km} \mathrm{~s}^{-1}$ for HD $11755,4.8 \mathrm{~km} \mathrm{~s}^{-1}$ for HD 12648, $3.5 \mathrm{~km} \mathrm{~s}^{-1}$ for HD 24064 , and $3.6 \mathrm{~km} \mathrm{~s}^{-1}$ for $8 \mathrm{UMi}$. Based on the rotational velocities and the stellar radii, we derived the upper limits for the rotational period of 600.5 days for HD 11755, 97.0 days for HD 12648, 549.3 days for HD 24064, and 139.1 days for 8 UMi.

\subsection{Photometric variations}

The HIPPARcos photometry data obtained from December 1989 to February 1993 (HD 11755), from December 1989 to March 1993 (HD 12648), from December 1989 to February 1993 (HD 24064), and from February 1990 to 
B.-C. Lee (이병 철) et al.: Search for exoplanet around northern circumpolar stars

Table 1. Stellar parameters for the stars analyzed in the present paper.

\begin{tabular}{lccccc}
\hline \hline Parameters & HD 11755 & HD 12648 & HD 24064 & 8 UMi & Ref. \\
\hline$\alpha(\mathrm{J} 2000)$ & 015850.087 & 021859.654 & 035636.297 & 145648.353 & 1 \\
$\delta(\mathrm{J} 2000)$ & +730908.58 & +854410.22 & +740448.12 & +745403.34 & 1 \\
Spectral type & $\mathrm{G} 5$ & $\mathrm{G} 5$ & $\mathrm{~K} 0$ & $\mathrm{~K} 0$ & 1 \\
$m_{v}(\mathrm{mag})$ & 6.87 & 6.98 & 6.75 & 6.83 & 1 \\
HIPPARCos & 0.010 & 0.009 & 0.009 & 0.008 & 1 \\
$B-V(\mathrm{mag})$ & $1.216 \pm 0.008$ & $0.904 \pm 0.008$ & $1.513 \pm 0.008$ & $0.985 \pm 0.010$ & 1 \\
Parallax $(\mathrm{mas})$ & $4.27 \pm 0.42$ & $6.29 \pm 0.36$ & $3.72 \pm 0.44$ & $6.25 \pm 0.43$ & 2 \\
Distance $(\mathrm{pc})$ & $231.5 \pm 22.7$ & $158.4 \pm 9.0$ & $264.4 \pm 31.2$ & $159.1 \pm 11.0$ & 3 \\
Age $(\mathrm{Gyr})$ & $10.2 \pm 1.3$ & $4.5 \pm 1.0$ & $9.0 \pm 2.1$ & $1.7 \pm 0.2$ & $4^{a}$ \\
$T_{\text {eff }}(\mathrm{K})$ & $4312.5 \pm 5.0$ & $4835.8 \pm 7.5$ & $4052.5 \pm 22.5$ & $4847.4 \pm 7.5$ & 4 \\
{$[\mathrm{Fe} / \mathrm{H}]$} & $-0.74 \pm 0.02$ & $-0.57 \pm 0.02$ & $-0.49 \pm 0.06$ & $-0.03 \pm 0.02$ & 4 \\
$\log g$ & $1.67 \pm 0.03$ & $2.18 \pm 0.03$ & $1.44 \pm 0.11$ & $2.57 \pm 0.03$ & 4 \\
$R_{\star}\left(R_{\odot}\right)$ & $27.3 \pm 1.0$ & $9.2 \pm 0.6$ & $38.0 \pm 2.9$ & $9.9 \pm 0.4$ & $4^{a}$ \\
$M_{\star}\left(M_{\odot}\right)$ & $0.9 \pm 0.1$ & $1.2 \pm 0.1$ & $1.0 \pm 0.1$ & $1.8 \pm 0.1$ & $4^{a}$ \\
$L_{\star}\left[L_{\odot}\right]$ & 145.72 & 45.01 & 352.90 & 55.94 & 3 \\
$v_{\text {rot }} \sin i\left(\mathrm{~km} \mathrm{~s}^{-1}\right)$ & 2.3 & 4.8 & 3.5 & 3.6 & $4^{b}$ \\
$P_{\text {rot }} / \sin i(\mathrm{days})$ & 600.5 & 97.0 & 549.3 & 139.1 & $4^{b}$ \\
$v_{\text {micro }}\left(\mathrm{km} \mathrm{s}^{-1}\right)$ & $1.50 \pm 0.04$ & $1.52 \pm 0.03$ & $1.53 \pm 0.09$ & $1.48 \pm 0.04$ & 4 \\
\hline
\end{tabular}

Notes. ${ }^{(a)}$ Derived using the online tool (http: //stevoapd.inaf.it/cgi-bin/param). ${ }^{(b)}$ See text.

References. (1) HIPPARCos; (2) van Leeuwen (2007); (3) Anderson \& Francis (2012); (4) this work.

March 1993 (8 UMi) were analyzed to find possible brightness variations that might be caused by the rotational modulation of large stellar spots. This database yields 149, 143, 162, and 111 measurements for HD 11755, HD 12648, HD 24064, and $8 \mathrm{UMi}$, respectively. All stars were photometrically constant down to an rms scatter of $0.008-0.010 \mathrm{mag}$. This corresponds to a variation over the time span of the observations of only $0.14 \%$, $0.13 \%, 0.13 \%$, and $0.12 \%$ for the four stars, respectively.

\subsection{Chromospheric activity}

Since the first discovery of emission lines in the core of the Ca II absorption feature (Eberhard \& Schwarzschild 1913), the EW variations of the Ca II $\mathrm{H}$ have been frequently used as a chromospheric activity indicator. The Ca II emission feature at the line center implies that the source function in the chromosphere is greater than in the photosphere. This is common in cool stars and is directly connected to the convective envelope and magnetic activity. Stellar activity can derive RV variations that can mask or even mimic the RV signature of orbiting planetary companions. Figure 1 shows the $\mathrm{Ca}$ II $\mathrm{H}$ line region of the six BOES spectra, and the chromospheric active star HD 201901 (K5 V) and the Sun (G2 V) are shown for comparisons. The star $8 \mathrm{UMi}$ lacks a prominent core emission feature in $\mathrm{Ca}$ II $\mathrm{H}$, and two stars, HD 11755 and HD 12648, are chromospherically modest. Unfortunately, the spectrum is not clear enough to resolve the emission feature for HD 24064. There may be a slight central emission in HD 24064, indicating a low level of stellar activity.

To clear up the ambiguity over chromospheric activity, we also measured variations in $\mathrm{H}_{\alpha}$ EW for the sample. The EW variations in the $\mathrm{H}_{\alpha}$ line are frequently used as the chromospheric activity indicator, along with $\mathrm{Ca}$ II $\mathrm{H}$ line (Montes et al. 1995; Kürster et al. 2003; Lee et al. 2012; Hatzes et al. 2015). We measured the EW using a band pass of $\pm 1.0 \AA$ centered on the core of the $\mathrm{H}_{\alpha}$ line to avoid nearby blending lines (i.e., ATM $\mathrm{H}_{2} \mathrm{O}$ 6561.1, Ti I 6561.3, Na II 6563.9, and ATM $\mathrm{H}_{2} \mathrm{O} 6564.2 \AA$ ). The individual chromospheric activity

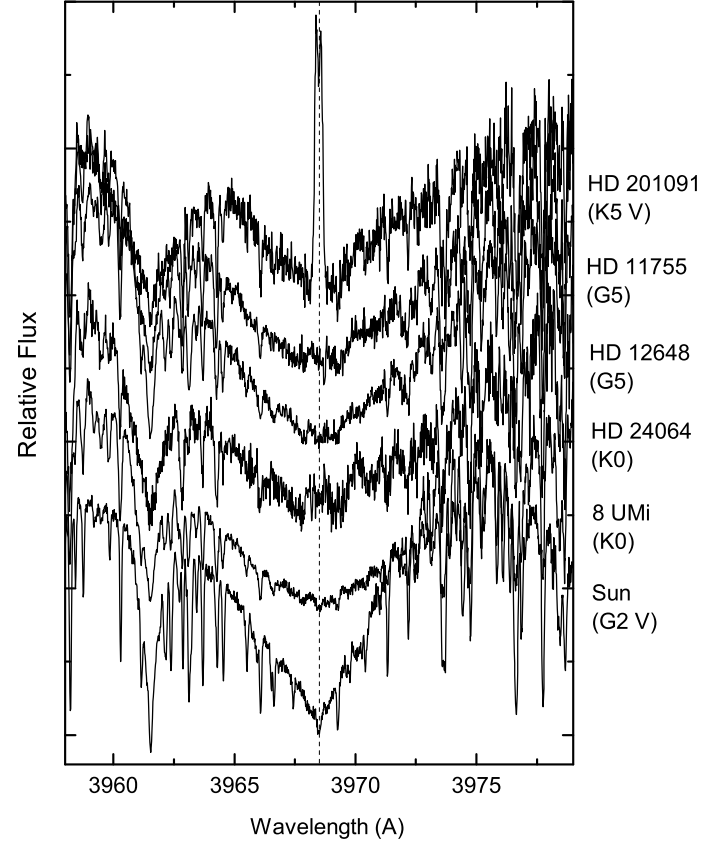

Fig. 1. Ca II H line cores for our program stars including the chromospheric active star HD 201091 and the Sun. There are no core reversals in the center of the $\mathrm{Ca}$ II H line for three stars except HD 24064. The vertical dotted line indicates the center of the $\mathrm{Ca}$ II $\mathrm{H}$ regions.

measurements of the $\mathrm{H}_{\alpha}$ line in Lomb-Scargle (L-S) periodograms are shown in the third panel of Figs. 4, 7, 10, and 13.

\subsection{Bisector variations}

Stellar rotational modulations by inhomogeneous surface features can create variable asymmetries in the spectral line profiles (Queloz et al. 2001). The differential RV measurements between 
Table 2. Orbital parameters for four exoplanets.

\begin{tabular}{lcccc}
\hline \hline Parameter & HD $11755 \mathrm{~b}$ & HD 12648 b & HD 24064 b & 8 UMi b \\
\hline$P($ days $)$ & $433.7 \pm 3.2$ & $133.6 \pm 0.5$ & $535.6 \pm 3.0$ & $93.4 \pm 4.5$ \\
$T_{\text {periastron }}(\mathrm{JD})$ & $2457018.2 \pm 14.0$ & $2452324.7 \pm 57.6$ & $2455278.3 \pm 11.8$ & $2454108.5 \pm 22.8$ \\
$K\left(\mathrm{~m} \mathrm{~s}^{-1}\right)$ & $191.3 \pm 10.2$ & $102.0 \pm 8.4$ & $251.0 \pm 9.3$ & $46.1 \pm 4.0$ \\
$e$ & $0.19 \pm 0.10$ & $0.04 \pm 0.16$ & $0.35 \pm 0.08$ & $0.06 \pm 0.18$ \\
$\omega(\mathrm{deg})$ & $155.3 \pm 12.6$ & $99.3 \pm 140.7$ & $250.8 \pm 6.3$ & $91.0 \pm 84.6$ \\
$m \sin i\left(M_{\text {Jup }}\right)$ & $6.5 \pm 1.0$ & $2.9 \pm 0.4$ & $9.4 \pm 1.3$ & $1.5 \pm 0.2$ \\
$a(\mathrm{AU})$ & $1.08 \pm 0.04$ & $0.54 \pm 0.02$ & $1.29 \pm 0.05$ & $0.49 \pm 0.03$ \\
$N_{\text {obs }}$ & 20 & 28 & 20 & 26 \\
rms $\left(\mathrm{m} \mathrm{s}^{-1}\right)$ & 27.7 & 29.8 & 34.5 & 17.2 \\
\hline
\end{tabular}

the high and low flux levels of the line profile are defined as a bisector velocity span (BVS $=V_{\text {top }}-V_{\text {bottom }}$ ). The changes in the spectral line bisector can also be quantified using the bisector velocity curvature $\left(\mathrm{BVC}=\left[V_{\text {top }}-V_{\text {center }}\right]-\left[V_{\text {center }}-V_{\text {bottom }}\right]\right)$. A BVS is simply the velocity difference in the bisectors of line widths between the top and bottom of the line profile, and a BVC is the difference in the velocity span of the upper half and the lower half of the bisector.

To search for variations in the spectral line shapes, we selected Ni I 6643.6 ̊ line as described in Hatzes et al. (2005) and Lee et al. (2013, 2014a), which is an unblended spectral feature with a high flux level and is located beyond the $I_{2}$ absorption region, so contamination should not affect our bisector measurements. We measured the bisector variations the profile between two different flux levels at 0.8 and 0.4 of the central depth as the span points, thereby avoiding the spectral core and wing where errors of the bisector measurements are large. The individual measurements for line bisectors are shown in the bottom panel of Figs. 4, 7, 10, and 13 (see the text).

\section{Radial velocity variations and their origins}

To search for periodicity in observed RV time series data, we performed the L-S periodogram analysis (Scargle 1982). This is a useful tool for investigating long-period variations for unequally spaced data. The resulting RV measurements are listed in Tables 3-6.

\section{1. $H D 11755$}

Twenty BOES spectra observations for HD 11755 spanning five years have been obtained, as shown in Fig. 2 and Table 3. The observations span four full orbital periods. The L-S periodogram of the RV measurements for HD 11755 shows a significant peak at a period of 433.7 days (top panel in Fig. 4). The L-S power of this peak corresponds to a false alarm probability (FAP) of $<1 \times$ $10^{-3}$, adopting the procedure described in Cumming (2004). We found that a semi-amplitude $K=191.3 \pm 10.2 \mathrm{~m} \mathrm{~s}^{-1}$ and an eccentricity $e=0.19 \pm 0.10$ for a Keplerian orbital fit. The rms of the RV residuals to the Keplerian orbital fit is $27.7 \mathrm{~m} \mathrm{~s}^{-1}$. We calculated the secondary period in the residual for HD 11755 , and it shows no significant peak (dashed line in the top panel in Fig. 4). Adopting a mass of $0.9 \pm 0.1 M_{\odot}$ for HD 11577, we fit the companion mass $m \sin i=6.5 \pm 1.0 M_{\text {Jup }}$ at a distance of $1.08 \pm 0.04$ au from the host star.

The HIPPARCos photometric data were analyzed, which shows no correlations with RV variations to check for the stellar brightness variations on HD 11755 (second panel in Fig. 4). The $\mathrm{H}_{\alpha}$ EW variations were examined to look for any RV fluctuations
Table 3. RV measurements for HD 11755 from September 2010 to May 2015 using the BOES.

\begin{tabular}{crr|rrr}
\hline \hline $\begin{array}{c}\mathrm{JD} \\
-2450000\end{array}$ & $\begin{array}{r}\Delta \mathrm{RV} \\
\mathrm{m} \mathrm{s}^{-1}\end{array}$ & $\begin{array}{r} \pm \sigma \\
\mathrm{m} \mathrm{s}^{-1}\end{array}$ & $\begin{array}{c}\text { JD } \\
-2450000\end{array}$ & $\begin{array}{r}\Delta \mathrm{RV} \\
\mathrm{m} \mathrm{s}^{-1}\end{array}$ & $\begin{array}{r} \pm \sigma \\
\mathrm{m} \mathrm{s}^{-1}\end{array}$ \\
\hline 5455.3146 & 122.8 & 13.7 & 6552.2523 & -155.6 & 9.9 \\
5820.3073 & -11.4 & 10.5 & 6578.2070 & -163.0 & 12.7 \\
5961.0203 & 164.8 & 43.5 & 6616.9513 & -214.5 & 10.5 \\
6253.2048 & -58.5 & 16.8 & 6808.2184 & 184.0 & 20.3 \\
6258.1507 & -15.2 & 11.3 & 6922.0666 & 53.4 & 10.8 \\
6261.1406 & 28.9 & 14.1 & 6964.0179 & -23.7 & 14.0 \\
6261.1538 & 35.2 & 14.1 & 6965.2009 & 10.6 & 24.9 \\
6286.0018 & 71.8 & 13.1 & 7065.9562 & -122.2 & 14.6 \\
6288.1130 & 53.7 & 11.7 & 7159.2983 & 72.7 & 19.8 \\
6551.1367 & -147.7 & 13.0 & 7171.2660 & 113.8 & 18.1 \\
\hline
\end{tabular}

that would be induced by stellar activity. Figure 4 shows the L-S periodgram of the $\mathrm{H}_{\alpha}$ EW variations (third panel). No correlation was found between the $\mathrm{H}_{\alpha} \mathrm{EW}$ and the RV variations. This means that HD 11755 exhibited at most a modest chromospheric activity at the moment of observations. Finally, line bisectors were measured by two kinds of methods. No correlation was found between the RV and the BVS variations or between the RV and the BVC variations (bottom panel in Fig. 4). All Keplerian orbital elements are listed in Table 2.

\section{2. $H D 12648$}

Observations for HD 12648 with the BOES took place between September 2010 and May 2015. We gathered a total of 28 data points during five years (Fig. 5 and Table 4). We found that the primary RV variations were fit best with a Keplerian orbit with a period of $P=133.6 \pm 0.5$ days, a semi-amplitude of $K=$ $102.0 \pm 8.4 \mathrm{~m} \mathrm{~s}^{-1}$, and an eccentricity of $e=0.04 \pm 0.16$. The rms of the RV residuals to the Keplerian orbital fit is $29.8 \mathrm{~m} \mathrm{~s}^{-1}$. We calculated a secondary period in the residual for HD 12648 , and it shows no significant peak (dashed line in the top panel in Fig. 7). Assuming a mass $M_{\star}=1.2 \pm 0.1 M_{\odot}$ for HD 12648, the result implies that the companion mass is $m \sin i=2.9 \pm 0.4 M_{\text {Jup }}$ at a distance of $0.54 \pm 0.02$ au from the host star.

The L-S periodograms of the HIPPARCOS measurements, the $\mathrm{H}_{\alpha}$ EW variations, and line bisector variations for HD 12648 are shown in Fig. 7. They do not show any obvious correlations with the RV measurements. This suggests that RV variations are not caused by line-shape changes produced by rotational modulation of surface features but by the planetary companion. 
B.-C. Lee (이병 철) et al.: Search for exoplanet around northern circumpolar stars

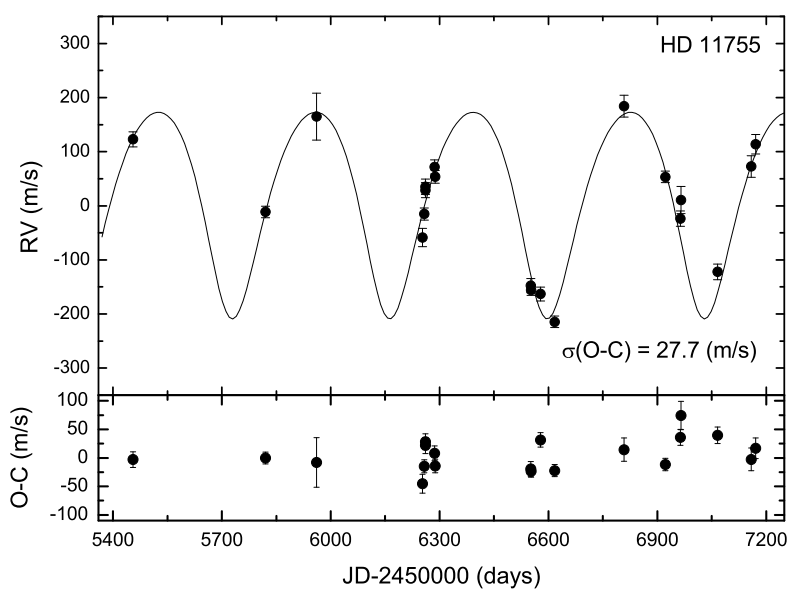

Fig. 2. RV measurements for HD 11755 from September 2010 to May 2015. Top panel: observed RVs for HD 11755, and the solid line is a Keplerian orbital fit with a period of 433.7 days, a semi-amplitude of $191.3 \mathrm{~m} \mathrm{~s}^{-1}$, and an eccentricity of 0.19 , yielding a minimum companion mass of $6.5 M_{\text {Jup }}$. Bottom panel: residual velocities remaining after subtracting the companion model from observations.

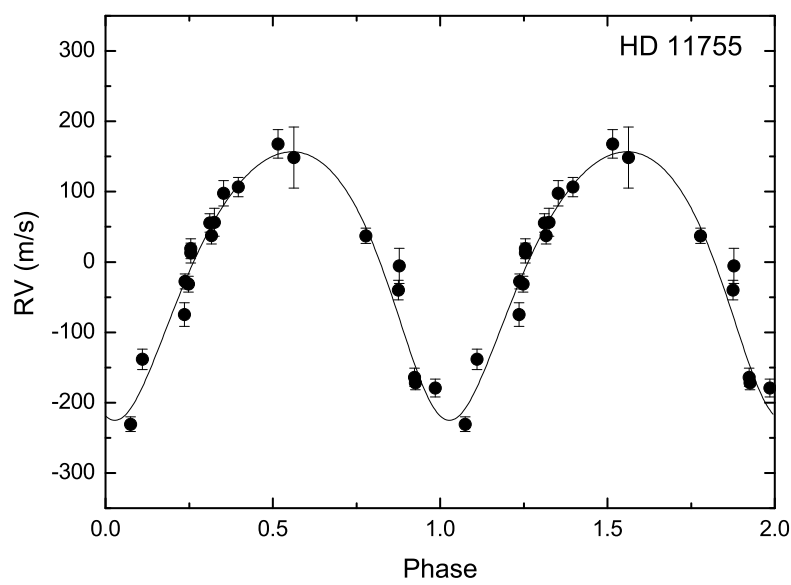

Fig. 3. RV variations for HD 11755 phased with the orbital period of 433.7 days.

\subsection{HD 24064}

Since February 2010, we have gathered 20 spectra for HD 24064 displaying in Fig. 8 and listed in Table 5. The observations span three and half orbital periods. The L-S periodogram of the RV measurements for HD 24064 shows a significant peak at a period of 535.6 days (top panel in Fig. 10). The L-S power of this peak corresponds to a FAP of $<1 \times 10^{-3}$. We found that semi-amplitude $K=250.8 \pm 6.3 \mathrm{~m} \mathrm{~s}^{-1}$ and eccentricity $e=$ $0.35 \pm 0.08$. After removing the main signal, the dispersion of the RV residuals is $34.5 \mathrm{~m} \mathrm{~s}^{-1}$, which is significantly higher than the RV precision for the RV standard star $\tau$ Ceti $\left(\sim 7 \mathrm{~m} \mathrm{~s}^{-1}\right)$ or the typical internal error of individual RV accuracy of $\sim 13.8 \mathrm{~m} \mathrm{~s}^{-1}$ for HD 24064. A periodogram of the RV residual, however, does not show any additional periodic signal as shown in Fig. 10 (top panel). Assuming a mass $M_{\star}=1.0 \pm 0.1 M_{\odot}$, we fit a companion mass $m \sin i=9.4 \pm 1.3 M_{\mathrm{Jup}}$ at a distance of $1.29 \pm 0.05 \mathrm{au}$ from HD 24064.

The L-S periodograms of the HIPPARCos measurements, the $\mathrm{H}_{\alpha}$ EW variations, and line bisector variations for HD 24064 are shown in Fig. 10. The $\mathrm{H}_{\alpha}$ EW does not show any obvious correlations with the RV measurements. We find a FAP of $18 \%$ for the peak at 89.8 days in the HIPPARCos measurements, and the

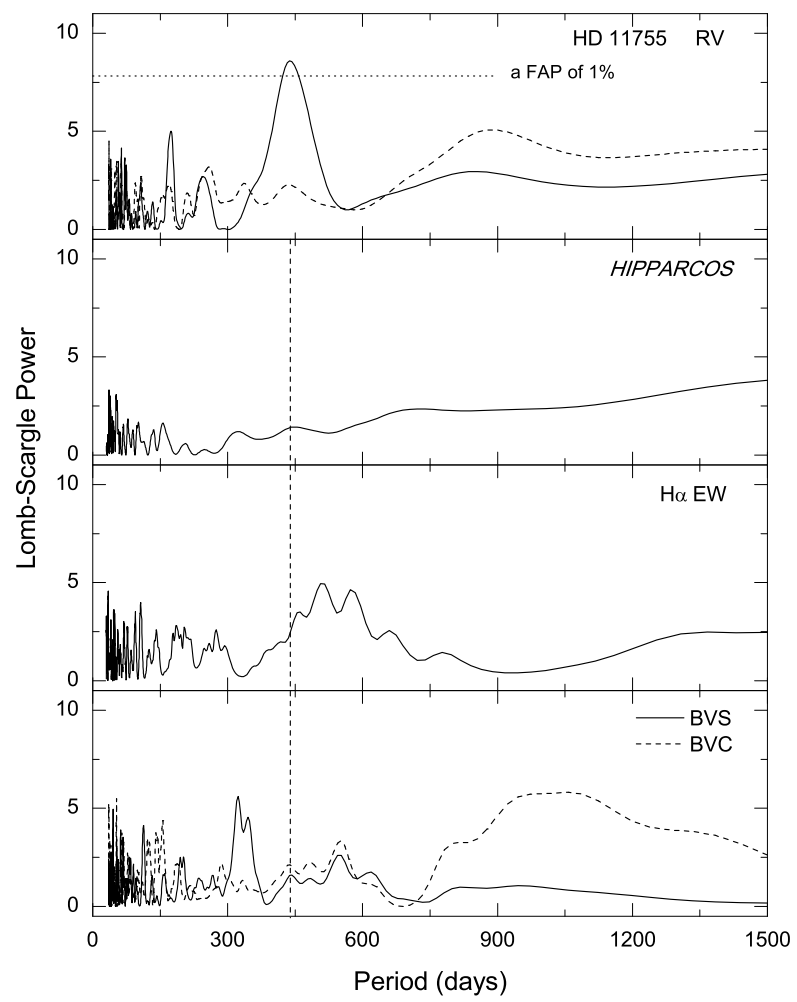

Fig. 4. L-S periodograms of the RV measurements, the HIPPARCos photometric measurements, the $\mathrm{H}_{\alpha} \mathrm{EW}$ variations, and the bisector variations for HD 11755 (top to bottom panel). The vertical dashed line indicates the location of the 433 day-period. Top panel: the solid line is the L-S periodogram of the RV measurements for five years, and the periodogram shows a significant power at a period of 433.7 days. The dashed line is the periodogram of the residuals after removing of the main period fit from the original data. The horizontal dotted line indicates a FAP threshold of $1 \times 10^{-2}(1 \%)$.

Table 4. RV measurements for HD 12648 from September 2010 to May 2015 using the BOES.

\begin{tabular}{crr|rrr}
\hline \hline $\begin{array}{c}\text { JD } \\
-2450000\end{array}$ & $\begin{array}{r}\Delta \mathrm{RV} \\
\mathrm{m} \mathrm{s}^{-1}\end{array}$ & $\begin{array}{r} \pm \sigma \\
\mathrm{m} \mathrm{s}^{-1}\end{array}$ & $\begin{array}{c}\mathrm{JD} \\
-2450000\end{array}$ & $\begin{array}{r}\Delta \mathrm{RV} \\
\mathrm{m} \mathrm{s}^{-1}\end{array}$ & $\begin{array}{r} \pm \sigma \\
\mathrm{m} \mathrm{s}^{-1}\end{array}$ \\
\hline 5455.3263 & -81.0 & 12.6 & 6808.0381 & 95.8 & 15.8 \\
5842.1888 & -89.3 & 12.4 & 6922.0962 & -22.3 & 13.9 \\
5933.0289 & -3.8 & 13.8 & 6965.2304 & 65.0 & 13.8 \\
5961.9263 & -113.9 & 14.5 & 6975.1407 & 91.3 & 13.3 \\
6259.0790 & 3.4 & 10.1 & 7024.8943 & -147.6 & 14.2 \\
6286.0988 & 118.0 & 15.3 & 7065.9045 & -12.1 & 16.4 \\
6288.1424 & 127.8 & 12.4 & 7065.9214 & -45.3 & 17.2 \\
6551.1848 & 29.0 & 14.1 & 7066.9422 & -47.8 & 14.0 \\
6578.2328 & 22.6 & 15.3 & 7068.0982 & -15.1 & 16.0 \\
6616.9759 & -100.0 & 10.9 & 7084.0117 & 57.5 & 18.5 \\
6620.1065 & -99.2 & 16.3 & 7084.0261 & 55.5 & 15.0 \\
6679.2195 & 76.2 & 15.8 & 7090.9466 & 64.3 & 16.4 \\
6711.3220 & 53.5 & 13.4 & 7111.0923 & 42.3 & 22.4 \\
6738.9917 & -75.6 & 14.6 & 7147.9716 & -50.6 & 18.7 \\
\hline
\end{tabular}

dominant peak of BVS is near 350 days with a FAP over $3 \%$, but they show no correlation to the RV. Even though there is a peak in the L-S periodogram of BVC roughly at 557 days, the FAP is $\sim 27 \%$, so it is not statistically meaningful. While this star shows some fluctuations in chromospheric activity, it is evident from Fig. 10 that stellar activities are uncorrelated with the RV measurements. Furthermore, the periodograms of 
Table 5. RV measurements for HD 24064 from February 2010 to May 2015 using the BOES.

\begin{tabular}{crr|rrr}
\hline \hline $\begin{array}{c}\mathrm{JD} \\
-2450000\end{array}$ & $\begin{array}{r}\Delta \mathrm{RV} \\
\mathrm{m} \mathrm{s}^{-1}\end{array}$ & $\begin{array}{r} \pm \sigma \\
\mathrm{m} \mathrm{s}^{-1}\end{array}$ & $\begin{array}{c}\text { JD } \\
-2450000\end{array}$ & $\begin{array}{r}\Delta \mathrm{RV} \\
\mathrm{m} \mathrm{s}^{-1}\end{array}$ & $\begin{array}{r} \pm \sigma \\
\mathrm{m} \mathrm{s}^{-1}\end{array}$ \\
\hline 5250.1474 & 68.2 & 20.2 & 6739.0180 & 209.4 & 18.0 \\
5842.2870 & 76.1 & 17.4 & 6808.2670 & 228.8 & 25.2 \\
5933.2105 & -81.4 & 17.8 & 6922.2232 & -39.5 & 17.7 \\
5959.0512 & -135.1 & 78.5 & 6965.9198 & -25.7 & 21.3 \\
6259.1815 & 233.8 & 12.7 & 7094.0278 & -251.9 & 21.0 \\
6287.0726 & 239.2 & 24.5 & 7129.9748 & -211.2 & 21.5 \\
6551.2761 & -231.4 & 21.0 & 7147.9867 & -120.5 & 20.3 \\
6582.2361 & -216.5 & 18.3 & 7155.9862 & -49.2 & 28.9 \\
6617.0197 & -69.6 & 12.9 & 7170.9927 & 96.3 & 33.3 \\
6714.0840 & 256.1 & 17.5 & 7171.2951 & 24.7 & 20.4 \\
\hline
\end{tabular}

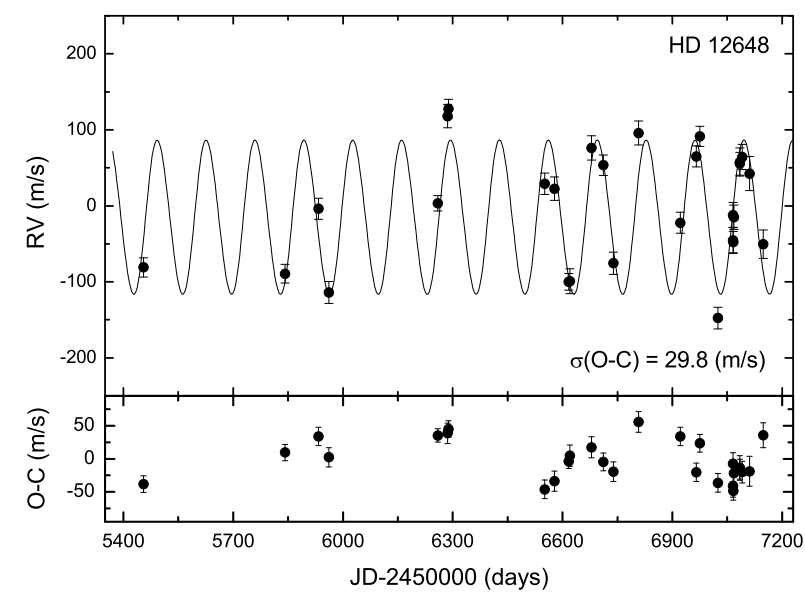

Fig. 5. RV measurements for HD 12648 from September 2010 to May 2015. Top panel: observed RVs for HD 12648, and the solid line is a Keplerian orbital fit with a period of 133.6 days, a semi-amplitude of $102.0 \mathrm{~m} \mathrm{~s}^{-1}$, and an eccentricity of 0.04 , yielding a minimum companion mass of $2.9 M_{\mathrm{Jup}}$. Bottom panel: residual velocities remaining after subtracting the companion model from observations.

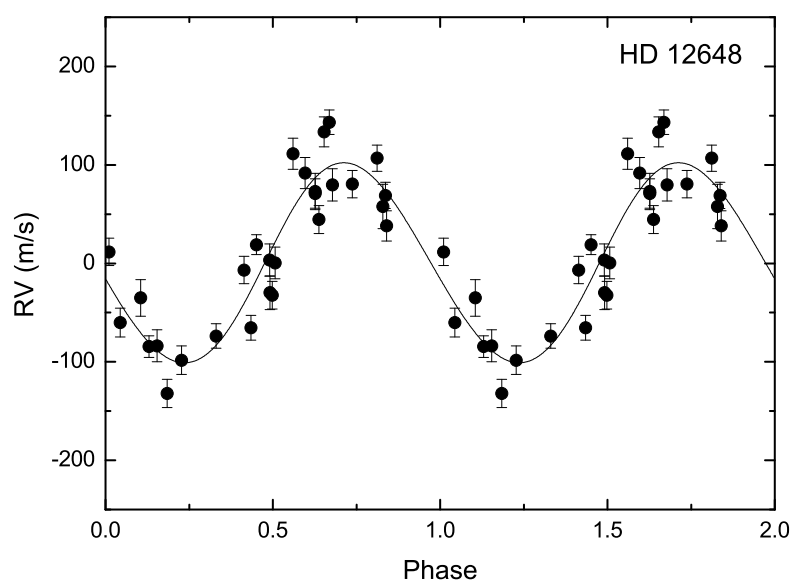

Fig. 6. RV variations for HD 12648 phased with the orbital period of 133.6 days.

the bisector also indicate no periodicity near the 535-day period of the planetary companion. These independent lines of evidence thus lead us to conclude that the RV variations observed in HD 24064 are not attributable to an intrinsic stellar process but to an orbiting giant planet.

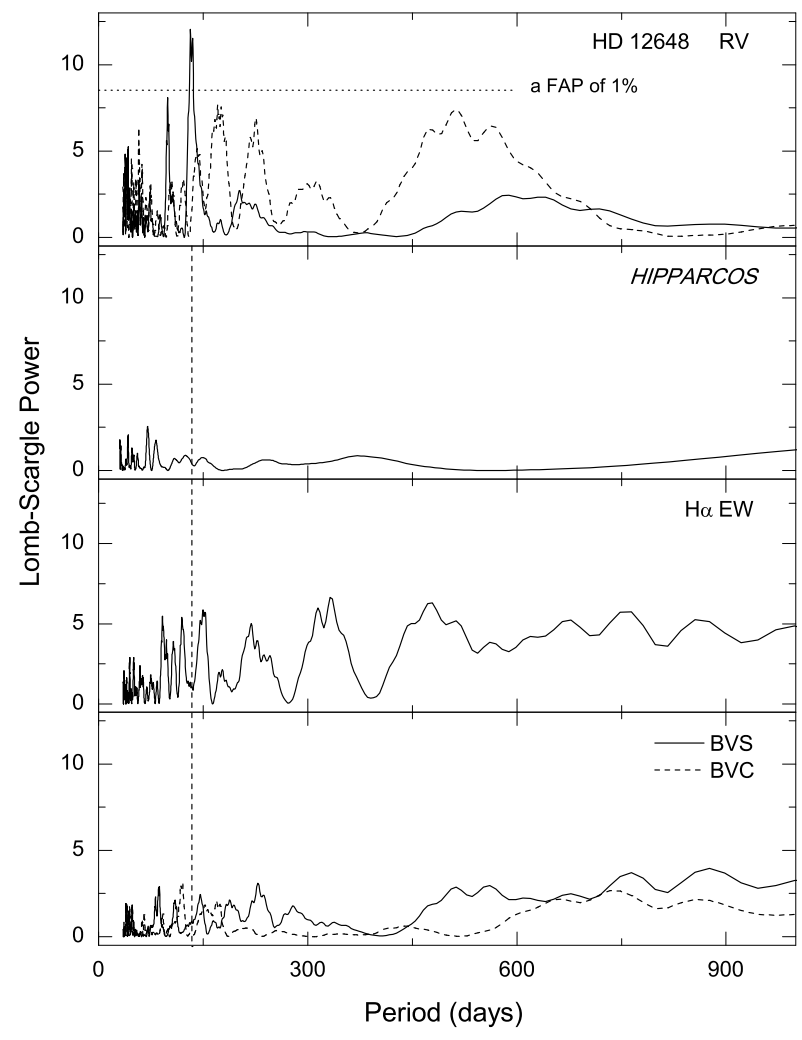

Fig. 7. L-S periodograms of the RV measurements, the HIPPARCOS photometric measurements, the $\mathrm{H}_{\alpha} \mathrm{EW}$ variations, and the bisector variations for HD 12648 (top to bottom panel). The vertical dashed line indicates the location of the period of 133 days. Top panel: the solid line is the L-S periodogram of the RV measurements for five years, and the periodogram shows a significant power at a period of 133.6 days. The dashed line is the periodogram of the residuals after removing of the main period fit from the original data. The horizontal dotted line indicates a FAP threshold of $1 \times 10^{-2}(1 \%)$.

\subsection{Ursae Minoris}

We obtained 26 BOES spectra for 8 UMi spanning five years (Fig. 11 and Table 6). The L-S periodogram of the RV measurements for $8 \mathrm{UMi}$ shows a significant peak at a period of $93.4 \pm 4.5$ days (top panel in Fig. 13). We found that semi-amplitude $K=46.1 \pm 4.0 \mathrm{~m} \mathrm{~s}^{-1}$ and eccentricity $e=0.06 \pm$ 0.18 for the Keplerian orbital fit. The rms of the RV residuals to the Keplerian orbital fit is $17.2 \mathrm{~m} \mathrm{~s}^{-1}$. By adopting a stellar mass of $1.8 \pm 0.1 M_{\odot}$ for $8 \mathrm{UMi}$, we obtain a minimum companion mass of $1.5 \pm 0.2 M_{\text {Jup }}$ and a semi-major axis of $0.49 \pm 0.03 \mathrm{AU}$.

The HIPPARCos measurements, the $\mathrm{H}_{\alpha}$ EW variations, and line bisector variations for $8 \mathrm{UMi}$ show no correlations with the RV measurements. The lack of any significant peaks in the L-S periodograms of chromospheric activity indicators shows that the RV measurements of $8 \mathrm{UMi}$ are caused by a planetary companion.

\section{Discussion}

Using the BOAO/BOES, we have begun a search for exoplanets around circumpolar stars in the northern hemisphere. All of our stars were surveyed for six years, and $\sim 16 \%$ of them revealed periodic RV variations. This is consistent with a prediction that about $18 \%$ of $\mathrm{F}, \mathrm{G}$, and $\mathrm{K}$ stars harbor a planet or candidate (Cumming et al. 2008). 
B.-C. Lee (이병철) et al.: Search for exoplanet around northern circumpolar stars

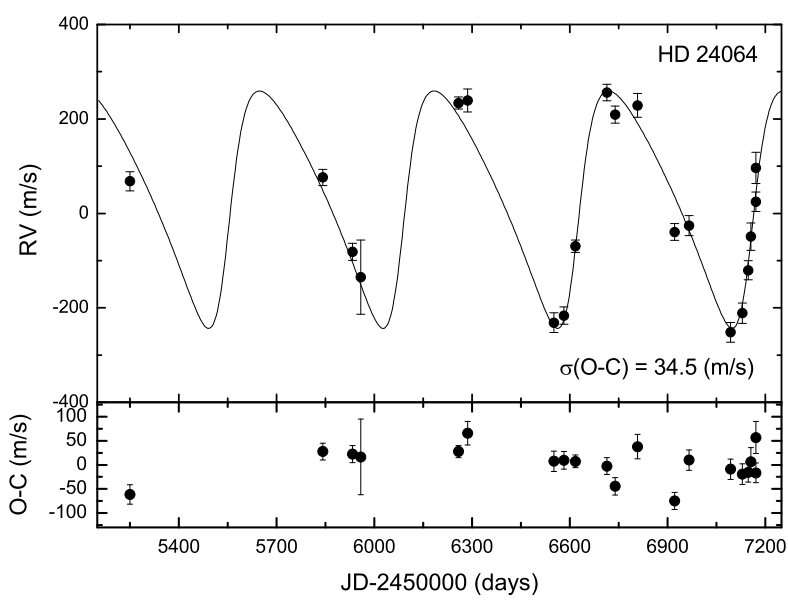

Fig. 8. RV measurements for HD 24064 from February 2010 to May 2015. Top panel: observed RVs for HD 24064, and the solid line is a Keplerian orbital fit with a period of 535.6 days, a semi-amplitude of $251.0 \mathrm{~m} \mathrm{~s}^{-1}$, and an eccentricity of 0.35 , yielding a minimum companion mass of $9.4 M_{\mathrm{Jup}}$. Bottom panel: residual velocities remaining after subtracting the companion model from observations.

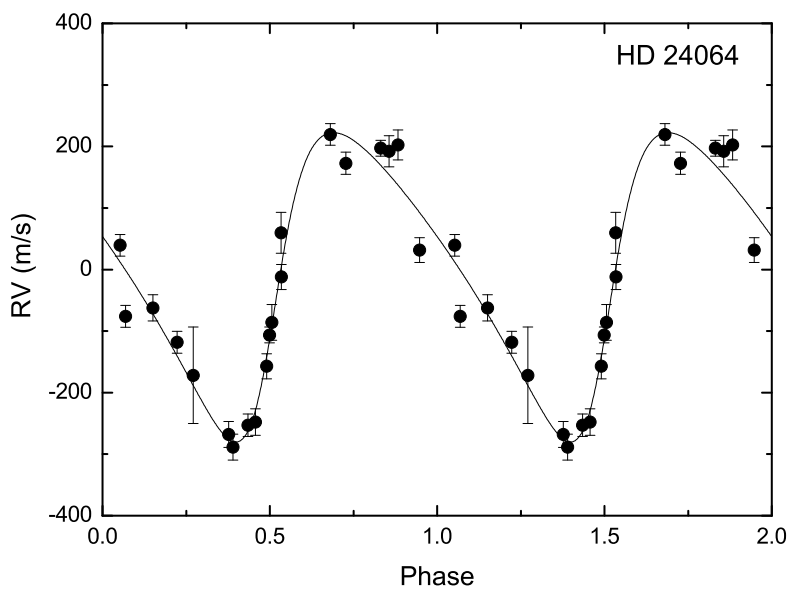

Fig. 9. RV variations for HD 24064 phased with the orbital period of 535.6 days.

We found periodic RV variations in HD 11755, HD 12648, HD 24064, and 8 UMi. They are evolved stars that are currently in the giant stage with stellar classifications of G0 and K0. Since most giants have intrinsic RV variations of several hundred days (Hekker et al. 2008), it is necessary to critically examine the planet hypothesis for these stars. Generally, most of them exhibit pulsations and/or surface activities with RV variabilities on different time scales. To establish the origin of the pure RV variabilities, some relevant analyses were carried out: rotational periods, photometric data, $\mathrm{Ca}$ II $\mathrm{H}$ line inspection, $\mathrm{H}_{\alpha} \mathrm{EW}$ measurements, and line bisector measurements.

The simplest test is to obtain the maximum rotational period. Based on the rotational velocities and the stellar radii, we derived the upper limits for the rotational period of 600.5 days for HD 11755, 97.0 days for HD 12648, 549.3 days for HD 24064, and 139.1 days for $8 \mathrm{UMi}$. Of these, because the RV period of HD 12648 (133 days) is longer than the upper limit of the rotational period of 97 days, observed RV variations in HD 12648 cannot be associated with the stellar rotation. Although the other three show no strong correlations between the RV variations and rotational periods, we cannot exclude the rotational modulations. We just note that the detected RV periods can be compatible with

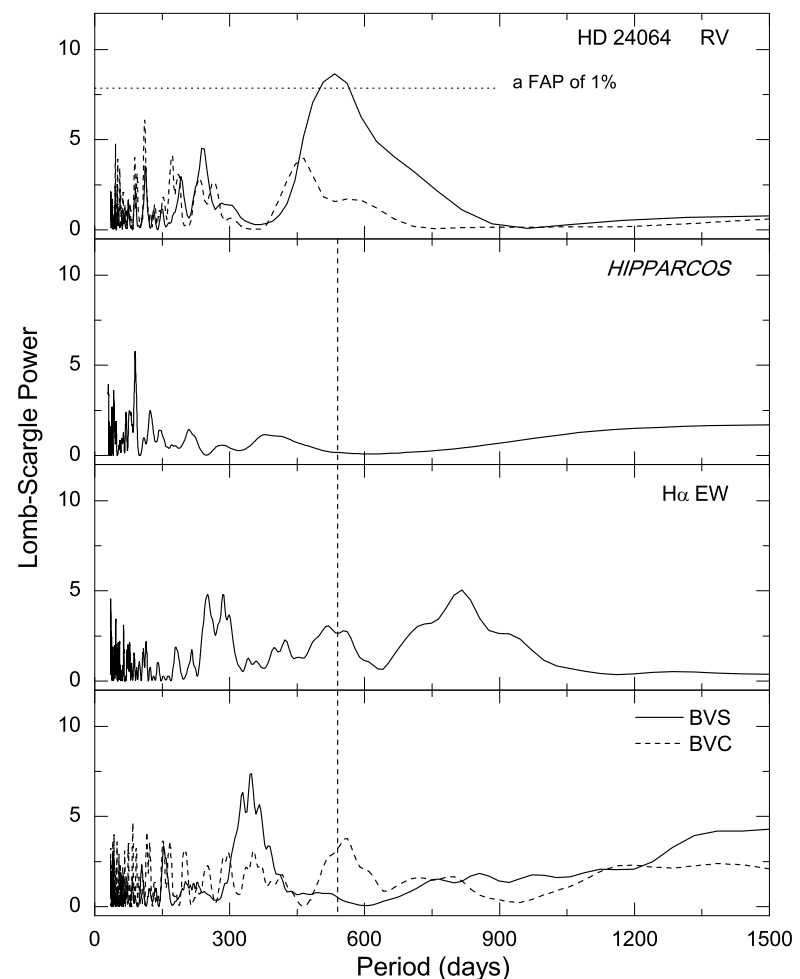

Fig. 10. L-S periodograms of the RV measurements, the HIPPARCos photometric measurements, the $\mathrm{H}_{\alpha} \mathrm{EW}$ variations, and the bisector variations for HD 24064 (top to bottom panel). The vertical dashed line indicates the location of the period of 535 days. Top panel: the solid line is the L-S periodogram of the RV measurements for five years, and the periodogram shows a significant power at a period of 535.6 days. The dashed line is the periodogram of the residuals after removing of the main period fit from the original data. The horizontal dotted line indicates a FAP threshold of $1 \times 10^{-2}(1 \%)$.

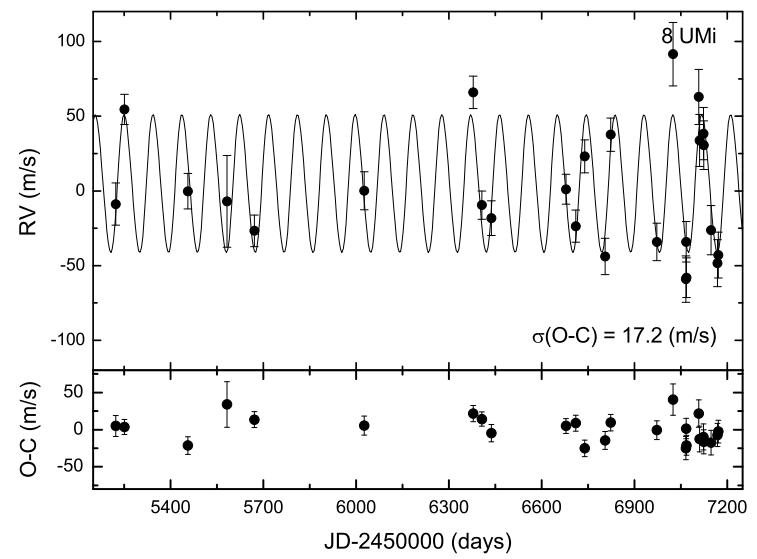

Fig. 11. RV measurements for 8 UMi from January 2010 to May 2015. Top panel: observed $\mathrm{RVs}$ for $8 \mathrm{UMi}$, and the solid line is a Keplerian orbital fit with a period of 93.4 days, a semi-amplitude of $46.1 \mathrm{~m} \mathrm{~s}^{-1}$, and an eccentricity of 0.06 , yielding a minimum companion mass of $1.5 M_{\mathrm{Jup}}$. Bottom panel: residual velocities remaining after subtracting the companion model from observations.

the rotational periods of the sample, even though that is not the most likely explanation.

However, the examination of the activity indicator $\mathrm{Ca}$ II $\mathrm{H}$ line does not show any obvious evidence of chromospheric activity for our sample. While a weak central emission may exist in the center of Ca II H line for HD 24064, which is 
Table 6. RV measurements for 8 UMi from January 2010 to May 2015 using the BOES.

\begin{tabular}{crr|rrr}
\hline \hline $\begin{array}{c}\mathrm{JD} \\
-2450000\end{array}$ & $\begin{array}{r}\Delta \mathrm{RV} \\
\mathrm{m} \mathrm{s}^{-1}\end{array}$ & $\begin{array}{r} \pm \sigma \\
\mathrm{m} \mathrm{s}^{-1}\end{array}$ & $\begin{array}{c}\text { JD } \\
-2450000\end{array}$ & $\begin{array}{r}\Delta \mathrm{RV} \\
\mathrm{m} \mathrm{s}^{-1}\end{array}$ & $\begin{array}{r} \pm \sigma \\
\mathrm{m} \mathrm{s}^{-1}\end{array}$ \\
\hline 5223.2931 & -11.1 & 14.2 & 6739.0549 & 20.8 & 11.1 \\
5251.3004 & 52.3 & 10.1 & 6805.0734 & -46.2 & 12.1 \\
5456.0690 & -2.5 & 11.8 & 6823.0469 & 35.3 & 11.1 \\
5582.4015 & -9.3 & 30.7 & 6972.3627 & -36.4 & 12.5 \\
5671.1018 & -29.0 & 10.6 & 7025.2594 & 89.2 & 21.2 \\
6026.2024 & -2.2 & 12.7 & 7066.1797 & -61.3 & 15.5 \\
6379.0953 & 63.6 & 10.9 & 7067.1929 & -36.4 & 13.6 \\
6407.1638 & -11.8 & 9.5 & 7068.1852 & -60.4 & 13.3 \\
6437.2342 & -20.6 & 11.6 & 7108.2615 & 60.6 & 18.4 \\
6679.2430 & -1.2 & 10.0 & 7111.1635 & 31.5 & 17.4 \\
6710.2830 & -25.9 & 10.7 & & & \\
\hline
\end{tabular}

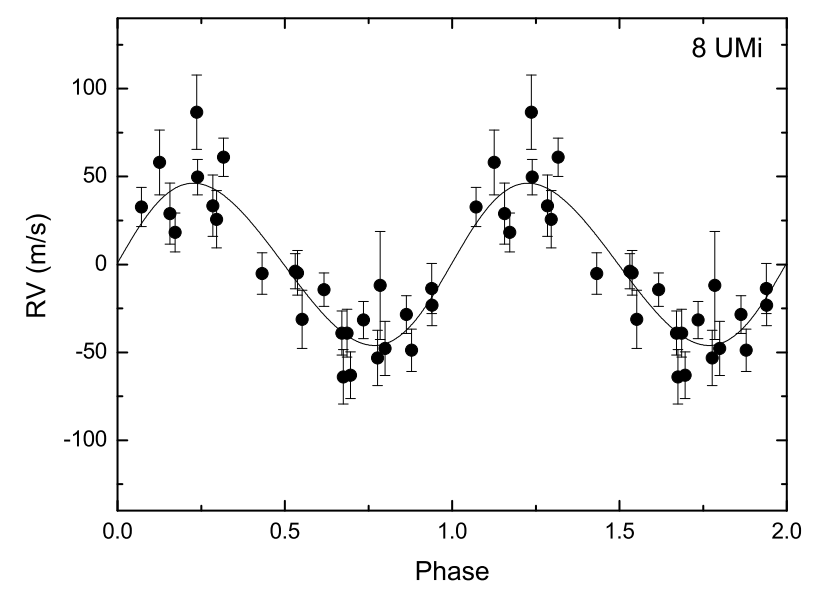

Fig. 12. RV variations for $8 \mathrm{UMi}$ phased with the orbital period of 93.4 days.

not strong enough to warrant the presence of the chromospheric active. From another measurements of chromospheric activity ( $\mathrm{H}_{\alpha} \mathrm{EW}$ variations), no significant peaks are evident, even in HD 24064. We can thus exclude the possibility that the main periodic RV variations observed in HD 24064 are induced by stellar activity. We identified the HIPPARCos photometries and calculated periodograms. None of the stars show photometric variations related to the observed RV variations. Line bisector analysis is a fairly common technique for determining whether periodic RV variations are caused by the rotational modulation of stellar spots. Such an analysis has the advantage of being contemporaneous with the RV measurements. No traces of line bisector variations were found to be associated with the RV variations.

We thus conclude that giants HD 11755, HD 12648, HD 24064, and 8 UMi host planetary companions with periods of 433, 133, 535, and 93 days and minimum masses of 6.5, 2.9, 9.4, and $1.5 M_{\text {Jup }}$, respectively. Figure 14 shows the distribution of the stellar radii versus the declinations of the exoplanets discovered so far. HD $12648 \mathrm{~b}$ becomes the exoplanet detected in the highest declination of $85.7^{\circ}$ so far, and HD $24064 \mathrm{~b}$ corresponds to an exoplanet found around one of the largest stars. The planets discovered in five of the six stars larger than HD 24064 were reported in another exoplanet survey program using $1.8 \mathrm{~m}$ telescope at BOAO (Lee et al. 2013, 2014b; Hatzes et al. 2015).

In summary, we found four new exoplanets around giant stars by expanding the exoplanet survey region to the north

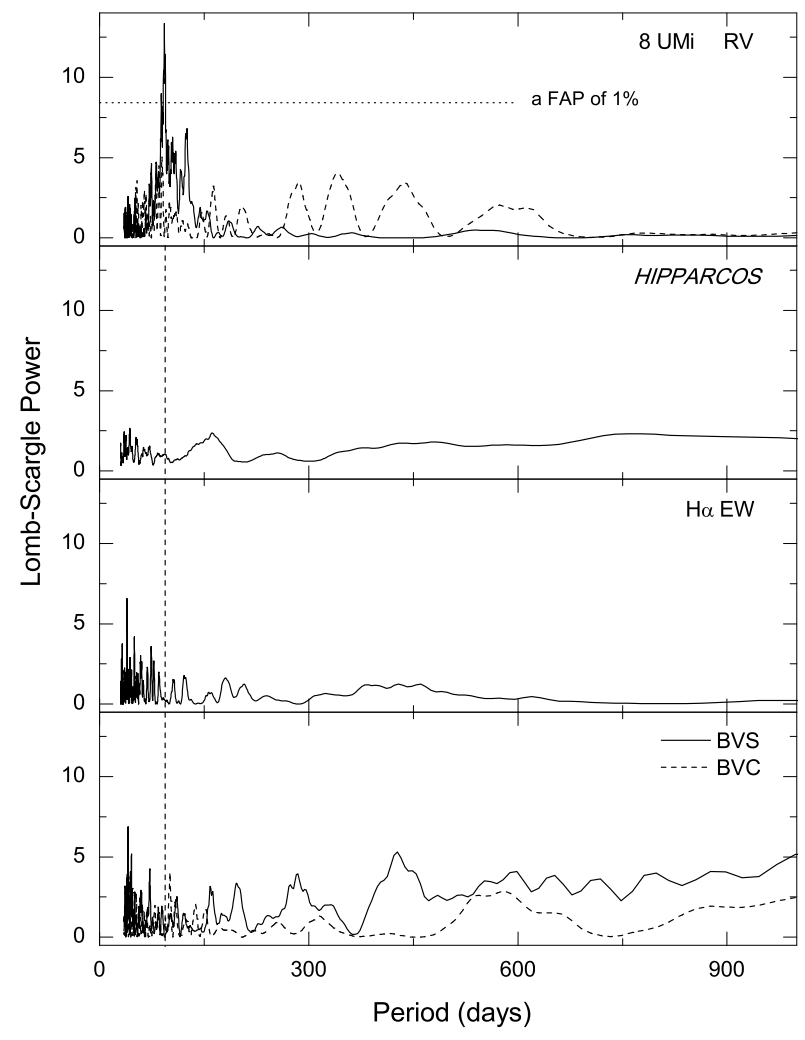

Fig. 13. L-S periodograms of the RV measurements, the HIPPARCos photometric measurements, the $\mathrm{H}_{\alpha} \mathrm{EW}$ variations, and the bisector variations for $8 \mathrm{UMi}$ (top to bottom panel). The vertical dashed line shows the location of the period of 93 days. Top panel: the solid line is the $\mathrm{L}-\mathrm{S}$ periodogram of the RV measurements for five years, and the periodogram shows a significant power at a period of 93.4 days. The dashed line shows the periodogram of the residuals after removing of the main period fit from the original data. The horizontal dotted line indicates a FAP threshold of $1 \times 10^{-2}(1 \%)$.

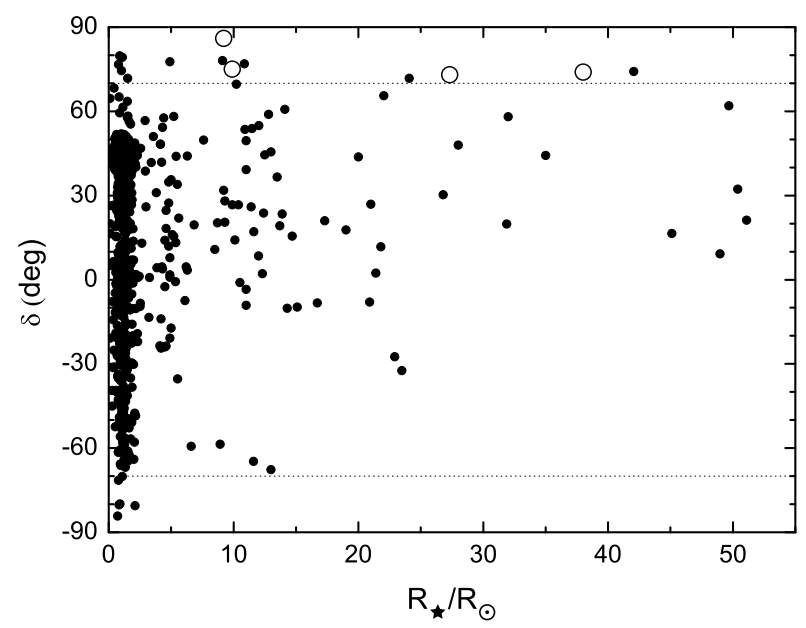

Fig. 14. Distribution of planetary companions between the stellar radii and declinations as of July 2015. Closed circles are known exoplanets, and open circles denote the locations of new four exoplanets HD 12648, $8 \mathrm{UMi}, \mathrm{HD}$ 11755, and HD 24064 (left to right). The horizontal dotted lines indicate a declination of $\pm 70^{\circ}$.

pole region (the declination $\delta \geq 70$ ). About 20 exoplanets (corresponding to $\sim 1 \%$ of the total) have been found around the pole region so far. Interestingly, nearly half have been found around giant stars. These discoveries will be important 
in understanding the planet formation around giant stars and the effect of stellar evolution on planet formation. Additional observations including the south pole region can confirm the statistical characteristics of these planetary systems.

Acknowledgements. B.C.L., J.W.L., C.U.L., and S.L.K. acknowledge partial support by the KASI (Korea Astronomy and Space Science Institute) grant 20151-850-04. Support for MGP was provided by the National Research Foundation of Korea to the Center for Galaxy Evolution Research (No. 2012-0027910). This research made use of the SIMBAD database, operated at the CDS, Strasbourg, France. We thank the developers of the Bohyunsan Observatory Echelle Spectrograph (BOES) and all staff of the Bohyunsan Optical Astronomy Observatory (BOAO).

\section{References}

Anderson, E., \& Francis, C. 2012, Astron. Lett., 38, 331

Anglada-Escudé, G., Arriagada, P., Vogt, S. S., et al. 2012, ApJ, 751, L16

Bonfils, X., Forveille, T., Delfosse, X., et al. 2005, A\&A, 443, L15

Bonfils, X., Lo Curto, G., Correia, A. C. M., et al. 2013, A\&A, 556, A110

Bowler, B. P., Liu, M. C., Shkolnik, E. L., \& Tamura, M. 2015, ApJS, 216, 7

Bressan, A., Marigo, P., Girardi, L., et al. 2012, MNRAS, 427, 127

Butler, R. P., Marcy, G. W., Williams, E., et al. 1996, PASP, 108, 500

Cumming, A. 2004, MNRAS, 354, 1165

Cumming, A., Butler, R. P., Marcy, G. W., et al. 2008, PASP, 120, 531

da Silva, L., Girardi, L., Pasquini, L., et al. 2006, A\&A, 458, 609

Döllinger, M. P., Hatzes, A. P., Pasquini, L., et al. 2007, A\&A, 472, 649

Eberhard, G., \& Schwarzschild, K. 1913, ApJ, 38, 292

Endl, M., Cochran, W. D., Tull, R. G., \& MacQueen, P. J. 2003, AJ, 126, 3099
ESA 1997, VizieR Online Data Catalog, I/239

Frink, S., Mitchell, D. S., Quirrenbach, A., et al. 2002, ApJ, 576, 478

Han, I., Kim, K.-M., Lee, B.-C., et al. 2007, Publ. Korean Astron. Soc., 22, 75

Han, I., Lee, B. C., Kim, K. M., et al. 2010, A\&A, 509, A24

Hatzes, A. P., Guenther, E. W., Endl, M., et al. 2005, A\&A, 437, 743

Hatzes, A. P., Cochran, W. D., Endl, M., et al. 2015, A\&A, 580, A31

Hekker, S., Snellen, I. A. G., Aerts, C., et al. 2008, A\&A, 480, 215

Howard, A. W., Marcy, G. W., Fischer, D. A., et al. 2014, ApJ, 794, 51

Jørgensen, B. R., \& Lindegren, L. 2005, A\&A, 436, 127

Kim, K.-M., Han, I., Valyavin, G. G., et al. 2007, PASP, 119, 1052

Kürster, M., Endl, M., Rouesnel, F., et al. 2003, A\&A, 403, 1077

Kurucz, R. L. 1992, The Stellar Populations of Galaxies, 149, 225

Lada, C. J. 2006, ApJ, 640, L63

Lee, B.-C., Han, I., Park, M.-G., et al. 2012, A\&A, 543, A37

Lee, B.-C., Han, I., \& Park, M.-G. 2013, A\&A, 549, A2

Lee, B.-C., Han, I., Park, M.-G., et al. 2014a, J. Korean Astron. Soc., 47, 69

Lee, B.-C., Han, I., Park, M.-G., et al. 2014b, A\&A, 566, A67

Montes, D., de Castro, E., Fernandez-Figueroa, M. J., \& Cornide, M. 1995, A\&AS, 114, 287

Queloz, D., Henry, G. W., Sivan, J. P., et al. 2001, A\&A, 379, 279

Reid, I. N., Gizis, J. E., \& Hawley, S. L. 2002, AJ, 124, 2721

Sato, B., Ando, H., Kambe, E., et al. 2003, ApJ, 597, L157

Scargle, J. D. 1982, ApJ, 263, 835

Setiawan, J., Hatzes, A. P., von der Lühe, O., et al. 2003, A\&A, 398, L19

Takeda, Y. 1995, PASJ, 47, 287

Takeda, Y., Ohkubo, M., Sato, B., Kambe, E., \& Sadakane, K. 2005, PASJ, 57, 27

Takeda, Y., Sato, B., \& Murata, D. 2008, PASJ, 60, 781

Valenti, J. A., Butler, R. P., \& Marcy, G. W. 1995, PASP, 107, 966

van Leeuwen, F. 2007, A\&A, 474, 653

Wright, J. T., Marcy, G. W., Butler, R. P., \& Vogt, S. S. 2004, ApJS, 152, 261 